\title{
El cambio de nivel: Todo un desafío
}

\section{Lili Berger, Jennifer Jones}

\section{INTRODUCCIÓN}

El presente trabajo de investigación intenta poner de manifiesto nuestra preocupación por el pasaje de un nivel a otro en el sistema educativo, en especial el cambio que se produce entre el Nivel Inicial y la Educación Primaria. Es indudable que son muchos los factores que acompañan el cambio del niño de un nivel a otro, algunos de ellos inciden positivamente y otros lo hacen negativamente. No todos los niños viven este pasaje de la misma manera lo que incide en su adaptación e integración posterior.

Nos inquieta entender cómo se opera esta transición, los cambios que se producen en este pasaje y en qué medida se atienden las características, necesidades e intereses de los niños. En el ámbito de la educación se ha polemizado acerca de si el J ardín de Infantes debe en su última etapa preparar a los alumnos para el ingreso a primer año o si por lo contrario, ningún nivel es preparatorio del que le sigue ya que cada uno posee sus objetivos propios. Se ha comprobado que los niños que han visto facilitado su aprendizaje a través de ricas y variadas experiencias tempranas pueden utilizar más eficientemente las nuevas experiencias que se les presenten en el futuro.

La Educación Inicial en tanto «espacio de enseñanza y aprendizaje con objetivos y contenidos que le son propios», debe propiciar una oferta educativa de calidad para capitalizar el potencial de aprendizaje de los pequeños.

Hemos planteado tres interrogantes que resultan básicas en nuestro plan de investigación:

¿Qué características se observan en ambos niveles? ¿Qué similitudes y diferencias existen entre ellos? ¿Cuál es el grado de articulación entre los niveles?

En conocimiento de las características fundamentales del Nivel Inicial y Primaria analizamos el grado de articulación entre ambos niveles con la finalidad de acercar propuestas concretas tendientes a favorecer este pasaje.

Hemos estudiado las aportaciones que han realizado las teorías de aprendizaje que han contribuido a optimizar el proceso pedagógico en el ámbito de la Educación Inicial. Y es a través de la teoría epistemológica - genética de J ean Piaget, según Eric de Corte $(1990)^{1}$, que la psicología infantil se ha visto enriquecida por el estudio a fondo de las relaciones entre el aprendizaje y la maduración, el estudio cualitativo de las estructuras, etapas, pasos del desarrollo infantil, y por la fundamentada insistencia en el papel de la estimulación adecuada al niño con el objetivo de facilitar, promover, ayudar y desarrollar su propio proceso de maduración y desarrollo de la inteligencia.

Las relaciones entre el desarrollo cognitivo y ap rendizaje, de acuerdo a la concepción vigotskiana, muestra la influencia permanente del aprendizaje en la manera en que se produce el desarrollo cognitivo: un alumno que tenga más oportunidades de aprender que otro, no solo adquirirá más información, sino que logrará un mejor desarrollo cognitivo.

\section{Objetivos de LA INVESTIGACIÓN}

\section{Objetivo General}

Contribuir al acercamiento entre la currícula del Nivel Inicial y la Escuela Primaria, propiciando la continuidad y coordinación del proceso enseñanza - aprendizaje.

\section{Objetivos Específicos}

a) determinar las condiciones de aprendizaje en los dos niveles, b) comparar las condiciones emergentes de los respectivos niveles, c) difundir los resultados de nuestra investigación a nivel institucional con la finalidad de promover un plan de acción en forma conjunta. 
Conforme al interés de nuestro trabajo seleccionamos cuatro aspectos que dan marco al proceso educativo. Ellos son: aspecto locativo, distribución del tiempo, enfoque metodológico y aspecto social (vínculos).

\section{Diseño Metodolócico}

En la presente investigación realizamos un estudio cualitativo para comprender la conducta humana desde el propio marco de referencia, fundamentado en la realidad, bajo la óptica de una observación naturalista. Adoptamos un enfoque etnográfico, como método de investigación social, de carácter holístico.

Es a través de la etnografía educativa que accedimos a valiosos datos descriptivos de los contextos, actividades y creencias de los participantes en los escenarios educativos. Focalizando el estudio del proceso educativo, de la ecología del aula, la Institución y sus relaciones.

Como instrumentos metodológicos empleamos observaciones participantes y entrevistas semiestructuradas. Las observaciones fueron cotidianas, deliberadas, sistemáticas, espontáneas, específicas de una situación, con un enfoque inclusivo. Las observaciones fueron acompañadas de registros fotográfico. En las observaciones participantes obtuvimos datos a través de unidades de análisis -cuadernos y producciones de los niños.

Las entrevistas fueron estandarizadas no presecuencializadas. En cuanto al contenido de las preguntas fueron formuladas preguntas descriptivas, estructurales y de contraste. Se analizaron un total de 26 entrevistas ( 13 a docentes y 13 a alumnos). La colecta de datos fue realizada en dos instituciones privadas, una de ellas bilingüe y otra cuyo idioma empleado es el inglés, no siendo ésta la lengua materna.

Los sujetos de investigación seleccionados en ambas instituciones fueron:

a) grupos de niños pertenecientes al Nivel Inicial y de Primer año. No se preestableció un criterio de selección de los grupos, se trató de abarcar la mayor cantidad de grupos existentes dentro de la institución.

b) docentes de las distintas áreas, titulares de los grupos y con diferentes niveles de antigüedad docente.

c) Informante clave: secretaria docente y psicóloga.

\section{Resultados}

El análisis de los datos nos permitió categorizar los aspectos más relevantes de dichas variables.

En el aspec to locativo los niños del J ardín de Infantes sienten una gran seguridad y mucho placer por el ámbito del J ardín. La sala de clase es muy tenida en cuenta en lo que hace a la distribución de rincones claramente definidos. Las actividades se realizan en la sala o en espacios exteriores lo que permite diversificar los entornos y propiciar mayor independencia de los alumnos.

En la Escuela Primaria una marcada limitación del espacio dentro y fuera del salón de clase fue la constante. Un aumento significativo de las dimensiones de la planta física, el elevado número de ámbitos dificulta los desplazamientos por la inseguridad que representa para el niño cambiar su marco de referencia.

En lo que respecta al enfoque metodológico en elJ ardín de Infantes se parte de un currículum flexible, globalizante que deja margen a lo circunstancial, no a la improvisación. Se promueven actividades variadas planificadas con alternancia en su estilo y nivel de exigencia, permitiendo asimismo desarrollar tareas colectivas, en pequeños grupos y en forma individual. Las áreas de trabajo aparecen amalgamadas entre sí, tras la búsqueda permanente de significatividad.

Resulta claro que es el juego el principal vehículo de acción. Acompañado de variados recursos didácticos, mediante la utilización de multimedios, surgen diversas propuestas generadoras de múltiples estímulos.

En Primaria el curriculum está estructurado en base a asignaturas definidas en cada una de las áreas. A pesar de los cambios metodológicos motivados por los lineamientos curriculares del aprendizaje sistematizado de la Escuela Primaria, los niños se van adaptando progresivamente con un marcado deseo de aprender.

El aspecto social fue abordado en forma global, integrando los dos niveles. Se constató un vínculo muy estrecho entre las maestras y los alumnos.

Fue unánime la afirmación de la buena disposición de los alumnos frente a las demandas escolares. Se denotó una clara jerarquización del aspecto afectivo - emocional pues to de manifiesto en la relación docente - alumno. Fue natural observar en el J ardín de Infantes una relación de mayor dependencia de parte de los niños con respecto al adulto, en relación con lo observado en Primaria. 
En cuanto a la distribución del tiempo varía entre un intenso devenir de actividades dentro y fuera del salón de clase y en los talleres específicos, a una marcada dosificación del horario acompañado de una paulatina integración de actividades y propuestas nuevas.

\section{CONCLUSIONES}

\section{Entendemos que:}

- La articulación es un problema que afecta a los pequeños y demanda respuestas de los grandes, de las instituciones y del sistema educativo configurado en un momento histórico y social determinado. La idea de articulación promueve la generación de un puente educativo entre un nivel y otro pensando en el niño, jerarquizando lo relacional, con el firme propósito de evitar las bruscas rupturas que se producen como consecuencia de las marcadas diferencias entre un nivel y otro.

- La articulación entre las etapas demanda reformulaciones didácticas que se acerquen a la realidad y a las necesidades de los niños pequeños sin que esto implique un «preparar para» sino por el contrario «vivir el hoy», promoviendo el aprovechamiento del juego y de las experiencias directas como base para enriquecer y potenciar las capacidades naturales de los niños.

- El intercambio y la retroalimentación a nivel docente entre la maestra del J ardín de Infantes y la maestra del 1er. Año, permitirá cierto grado de continuidad sin «primarizar el J ardín y tampoco infantilizar la Escuela Primaria».

Percibimos que el Nivel Inicial es el que más acompaña y atiende las características de los niños de corta edad debido a que:

a) Orienta su práctica educativa al desarrollo integral del niño en la primera infancia.

b) Integra, orienta y fortalece a la familia como coeducadora.

c) Asiste al niño en su handicap y a su vez intenta potencializar al máximo su desarrollo.

d) Hace de la afectividad y de las relaciones vinculares el eje medular de la propuesta pedagógica.

e) Enfatiza un enfoque interdisciplinario, globalizante donde el juego aparece como un fin en sí mismo, como la expresión del esfuerzo que realiza el niño para adaptarse a la realidad.

f) Maneja criterio de flexibilidad en lo que hace a la distribución del tiempo, la programación y las pautas de evaluación.

g) Busca promover una atención personalizada donde el hacer vale por los procedimientos empleados, por los caminos abordados.

\section{A Modo De Cierre}

Es necesario plantear la gran responsabilidad que le compete a la Institución Educativa en la planificación, ejecución y evaluación del pasaje del J ardín de Infantes a ler. Año, así como también crear una atmósfera favorable al aprendizaje en donde el niño se sienta independiente, utilice su propia iniciativa en perseguir sus intereses, en decir lo que piensa, hacer preguntas, proponer ideas.

La Escuela debe tender a lograr un intercambio de información entre los docentes de cada nivel, aunar criterios pedagógicos para dar coherencia a la tarea educativa, así como también acompañar y continentar al niño en el tránsito, en el pasaje de un nivel a otro.

"LA EDUCACIÓN INFANTIL NO SE TRATA SOLO DE SABER MÁS COSAS, SINO DE VIVIR MÁS COSAS".

'De Corte, E. (1990), "Aprender en la Escuela con las nuevas tecnologías de Información" (Conferencia UCUDAL). 